\title{
5 COMMUNICATION INFRASTRUCTURE REQUIREMENTS IN A VE
}

\section{A. Luís Osório ${ }^{1 \bullet}$, Manuel M. Barata ${ }^{1}$, Pierre Gibon ${ }^{2}$ ${ }^{1}$ ESTEC, Estudos e Tecnologias da Informação, Portugal ${ }^{2}$ LICHEN Informatique, France}

\begin{abstract}
This chapter presents the general communication infrastructure requirements, considering the operation of enterprises in the context of Virtual Enterprises (VE). A VE is grounded on a distributed system based on a group of computers and software applications located in different companies that are supposed to exchange different information types. One important premise is the adoption of SME as the main target, what introduces constraints as service quality at low communication costs.
\end{abstract}

\section{INTRODUCTION}

The Virtual Enterprise (VE) paradigm is strongly dependent on communications. Enterprises belonging to a consortium structured around a VE business concept (Camarinha-Matos, 1997) are supposed to communicate widely and intensively, when executing their cooperation activities.

Communication can be done involving many communication languages and protocols. On the one hand, the Enterprise Resource Planning (ERP) and business applications have to exchange business documents and technical data among them. Furthermore, the communication among different enterprises should be done guaranteeing minimal communication costs, high availability and an internal enterprise communication equivalent security. The recent evolutions in this area point to a generalized exchange of information and the extension of the internal enterprise software tools to support distributed collaboration. The World Wide Web (WWW) or simply Internet is a paradigmatic example of this explosion (Macgregor, 1996), (Stross, 1997). Nowadays, there are a significant number of worldwide enterprises using this infrastructure to support some facets of their business. Evident benefits, such as the global information access, enlargement of the enterprise

\footnotetext{
- ESTEC Lda., TAGUSPARK, Edificios Tecnologias I, N. 21, 2780 Oeiras, Portugal,

Tel: +351-1-4220120, Fax: +351-1-4214090, lo@estec.pt
} 
visibility, reduction of the time necessary to do business, and others, are being understood by all business interlocutors. The synergy generated by the Internet services is forcing everyone to look at this new technology and to integrate it to support their business processes. One example of this tendency is the increasing level of e-mail addresses that are being used as a fast way to exchange information, not only private but also as a business support. Nevertheless, this boom brought a set of new problems to the communication infrastructure. The openness of the communication protocols forces the utilization of techniques to guarantee the privacy of communication channels and the establishment of authentication mechanisms to provide interlocutors credibility (Osório, 1997).

The main goal of this chapter is to present and discuss the communication infrastructure requirements for the virtual enterprise operation. Besides communication, privacy is also an important topic considering the necessary availability of a private network equivalent connecting virtual enterprise nodes and guaranteeing messages privacy and integrity and authentication of all enterprise members. The credibility of any communication infrastructure deeply relies on two technological aspects: the availability of the communication channels and its degree of privacy. The complexity of the Internet communication services (Jamsa, 1995), (Dowd, 1996) and the panoply of involved protocols, in addition to its open characteristic, create some expectations and concerns in enterprises managers. This chapter intends to clarify some important aspects of the Internet protocol suit arguing in favor of its adoption as PRODNET low level communication stack.

\section{COMMUNICATION REQUIREMENTS}

Communication among different computational processes or applications, produced by different application developers, adds complexities at different levels. Beyond a mandatory common information language/format, the physical distribution involves also security issues.

In an ascending classification complexity communication can be first considered among applications running in the same computer, second between applications running in different computers located in the same local networks (Intranets) and, finally, the communication among applications running in computers located in different Intranets. The last scenario is, in fact, the most complex for two main reasons: on one hand, whatever the communication mechanism is, it can fail; on the other hand, there are several security-related problems that contribute to the complexity of a distributed system (Tanenbaum, 1994), (Coulouris, 1994). There are also some relevant aspects from the application side that constrain the characteristics that may be available in a communication infrastructure. From those characteristics the most relevant are:

- communication availability and quality;

- communication resources availability and communication costs;

- information security/privacy and integrity;

- authentication;

- date and time co-ordination;

- delivery report and receipt notification-legal compliance. 
In the following points some of these requirements are overviewed, considering the establishment of such a communication infrastructure.

\section{Communication availability and quality}

A key requirement for an enterprise information system is to present a uniform / integrated and complete / consistent information access and management.

Considering the fast information technology innovations occurred during the last decades, most of the SME are facing the challenge to create uniform views for their isolated sub-systems. The lack of a general framework to establish the enterprise information systems structure and operation rules has contributed to the development of enterprise applications with incompatible views to similar information items. This situation leads to the proliferation of several subinformation systems managed by specific applications, creating information duplication along the enterprise. This situation also creates added difficulties to the enterprise information managers, to guarantee the enterprise information consistency and a general information flow among different applications. From the communication point of view the enterprise applications need to share and exchange information items, independently of the location of the interoperable applications. To achieve this goal they need to communicate different information items, requiring the establishment of open languages and standard protocols.

Therefore, enterprises' communication needs range from the exchange of small messages up to huge files containing STEP product models, business documents in EDIFACT format, complex picture in GIF language, and many other information contents, formats or languages. The communication infrastructure might offer a set of communication services for the enterprise applications executing enterprise activities and running in different enterprise nodes.

The amount of information bulks must be compatible with the available communication resource bandwidth. It does not make sense to send a file containing about $10 \mathrm{MB}$ through a communication channel of $1 \mathrm{~KB} / \mathrm{s}$ as it will take about 3 hours. It is not always possible to provide a communication channel with enough bandwidth, using exclusively one communication resource. Techniques such as message compression, selection of alternative communication resources, and others should be used to minimize message transference times. Another important aspect when dealing with large information elements, like STEP files, is to provide an "intelligent" file exchange mechanisms in order to avoid systematic exchange restarting, leading to wasteful communication channel occupation.

\section{Communication resources availability and communication costs}

If a communication resource has a momentary failure, the need to find an alternative path relies on the message urgency.

It is important to define a set of recovery procedures, able to find alternative solutions depending on the urgency and responsibility of the message to be delivered. In front of situations where it is not possible at all to deliver the message, a local temporary storage (queuing) should be provided to enable later retries. 
The permanent availability of the communication facilities is not mandatory. As an example, when using e-mail to exchange an EDI message, is only necessary to access e-mail account at specific frequencies or dates according the commercial agreements established between the partners. However, depending on the urgency and responsibility associated to special messages, automatic recovery mechanisms should be provided to guarantee enough efficiency in message delivery.

\section{Information security/privacy and integrity}

Privacy is a key requirement for a communication infrastructure to be used to exchange enterprise business documents.

Privacy at different levels is mandatory to the success of any communication infrastructure that is based on any untrustworthy communication resources like Internet. The utilization of open protocols and public communication resources impose the implementation of mechanisms that guarantee, to the virtual enterprise, the same privacy available inside the enterprise. Furthermore, the communication infrastructure must guarantee message integrity regardless its content and size. Enterprise applications send messages considering that the communication stacks assume the integrity of the exchanged data. The EDIFACT standard allows only a few basic message integrity controls, aiming to validate file content integrity. The following two segment types are example of such features:

- UNT - message trailer segment with the number of segments contained within a message;

- UNZ - interchange final segment with the number of messages contained in the interchange.

However, this feature is deeply related with the accessibility to an EDI message when exchanged through a file-system file. Documents might flow with a total confidence on communication resource data integrity.

As far as privacy is concerned, the requirements to protect exchanged data depend on the classification of its contents. Most of the times there are no need to protect all the messages, but rather partial information items. As an example, an EDI/EDIFACT message transporting an invoice or an order may be delivered considering only partial encryption (Schneier, 1996) of relevant field values. In this context two strategies can be considered:

- full message encryption and signature;

- partial message encryption and signature.

At any moment, the decision about which option to adopt depends on partner's profile, considering the established agreements. Depending on the enterprise policy the applications may require the encryption and/or the signature of parts of a document to be delivered (Schneier, 1996), (Willmott, 1998).

\section{Authentication of the enterprise nodes}

When a business message is received, the identification of the originator is checked against identification elements that the network can provide. 
Based on the fact that the majority of EDI users exchange data through wellknown networks, this control is sufficient for standard commercial transactions. When using untrustworthy networks like Internet, other mechanisms are required in order to obtain a reasonable degree of confidence on message sender identification. It is the communication infrastructure responsibility to offer enterprise applications undoubted sender identification, providing that any tentative from an impostor to make business on the behalf of any enterprise is detected. This basic requirement is also related with the need for the establishment of commitments about the businessexchanged documents. Those commitments can involve the need for conflict resolution, for which would be necessary to prove, at both sides, the taken business initiatives, e.g., messages delivered and signed electronic receipts.

\section{Date and time co-ordination}

In most of the commercial transactions, the date and time is of primordial importance. When an enterprise sends an EDI message like ORDERS or DELFOR, there is a maximum time limit for the message to arrive to the destination.

In the commercial links established for streamlined production, this time can be reduced until a few hours. This is not properly a real time constraint, but it may have some influence when choosing the communication resource. As an example, the X.400 protocol allows message sending with associated optional time limits (a nondelivery report is supplied, if the message is not received within a given time). However, considering the utilization of Internet or any other communication resource without basic quality of service, is up to the communication infrastructure to manage the communication quality considering time constraints. Another requirement when dealing with enterprise nodes, where different time zones have to be considered on a global perspective, is the transparency that the communication infrastructure might present for such time/date differences.

\section{Delivery report and receipt notifications - legal compliance}

Delivery logs and message acknowledgements are mandatory facilities that might be available to the message sender.

They enable managers to track for errors, adopt recovery procedures, and to report the commercial exchange status. In fact, electronic business document flow needs to be logged, to allow posterior auditing in front of some exceptional situation. Legal constraints may require the electronic exchanged documents to be maintained for some time period (fixed number of years) for auditing purpose (taxes control). This aspect is of primordial importance since entrepreneurs have to trust/believe on all the available mechanisms to manage their enterprise business documents.

Another important factor in a communication infrastructure is to provide quality of communication services good enough to offer the confidence from all the involved people, starting from the entrepreneurs, to information system administrators and enterprise application users. At the enterprise application level there are other requirements besides the ones related with the communication infrastructure. The privacy of all the exchanged information also depends on how the enterprise users manage relevant information to maintain the system security like 
the cryptographic keys. However, these aspects are not addressed by the communication infrastructure.

\section{TOWARD A COMMUNICATION INFRASTRUCTURE}

During the last decades several research groups, standardization bodies and other initiatives have contributed to a global framework able to promote a general interoperability among heterogeneous subsystems.

Since long time researchers and developers have the perception that a general movement toward a general automation of enterprise processes can only be done joining efforts from different developers, converging to a common view of the emergent communication and computational technologies in this area. This topic presents the PRODNET II contribution to this trend, stressing the openness perspective of the PRODNET infrastructure and its ability to deal with several communication resources.

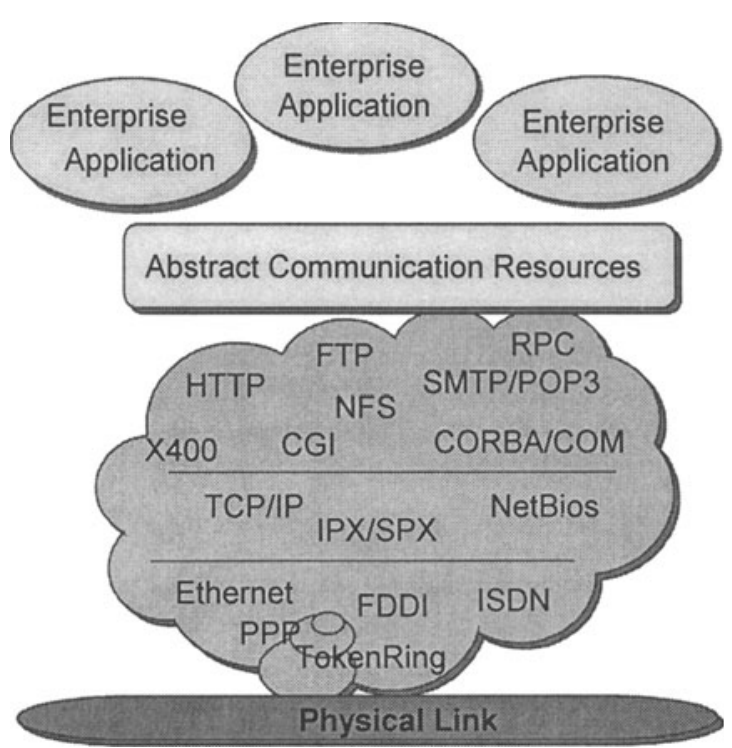

Figure 1 - Abstraction of different communication protocols (resources)

\section{The middleware and the business application level}

Since the earlier times of the network communication and computing developments one of the main concern is the difficulty to deal with a distributed system loosely connected through a local or wide network.

The networking faces always several availability problems, most of them related with the absence of a clear and homogeneous infrastructure connecting all subsystems. One factor that has contributed to this lack of quality in distributed systems, is the continuous evolution of the communication technologies at all levels. 
During the last decades many refinements to the evolving technologies have generated the emergence of new and incompatible technological solutions, leading to large investments when required to be up to date with the technology edge.

Due to the increasing adoption of computational technologies to automate different enterprise sectors, one problem that was always faced by information systems managers: how to exchange information among the heterogeneous enterprise applications, in order to create a virtual homogeneous enterprise information management system. The problem is worst when the goal is the interoperation between applications from different enterprises. Beyond the application information exchange problems, there is another complexity related with the information exchange among enterprises crossing different communication resources and computational systems. Ranging from simple file transfer to the emergent object/component information access, let uss consider some of the possibilities that exist to exchange information among distributed components. There are a large number of languages and protocols to support information exchange among distributed components:

- file exchange: the exchange can be performed manually (floppy, etc.), through File Transfer Protocol (FTP), file sharing (NFS - Network File System, etc.) or other means;

- e-mail, using the available protocols such as Simple Mail Transfer Protocol (SMTP), Multipart Internet Message Extensions (MIME) RFC 1521 embedded protocol providing transport facilities for multiple binary data types and Post Office Protocol (POP3) for clients supporting multimedia extensions;

- client-server paradigm: direct connections supported by protocols implemented above TCP/IP (DARPA protocols suit). Examples of this are: Remote Procedure Call (RPC), HyperText Transport Protocol (HTTP), etc. Several application level protocols are based on TCP/IP suit, since they present some added value concerning specific characteristics of the communication needs;

- object oriented communications based on object paradigm: the CORBA initiative proposes a framework providing objects to interact through an Object Request Broker (ORB). The Component Object Model (COM) proposed by Microsoft is another example of a similar infrastructure.

As a matter of fact, many solutions have been developed to provide applications interaction based on the client-server paradigm. However, the client-server model is not tailored to support the PRODNET communication infrastructure because a peer to peer interaction among enterprise nodes is required. There are however some features emerging from the Object Management Group (OMG) initiatives, that can greatly contribute to speed-up the software development and guarantee an extended interoperability among other enterprise subsystems.

\section{Generality of the communication infrastructures}

There are two perspectives on how to address a communication infrastructure to support the information exchange among enterprises. 
Some available market solutions point to the establishment of Private Virtual Networks (PVN), connecting different sites from the same enterprise (see Figure 2) or even different enterprises on a bilateral technological agreement. Components like intelligent routers, firewalls (Cheswick, 1996) and other communication subsystems are available to enable applications running at different sites to communicate safely, using the standard operating system communication services. An infrastructure is established to create a virtual network connecting all the enterprise sites, which offers secure communications among all the sites for any application using standard communication mechanisms. This PVN requires that any enterprise that adopts it have to acquire similar communication equipment to all the sites. Besides, if other enterprises are also involved in such VPN, they also have to adopt compatible technology. Even if some normalization degree exists, the emergence of new communication protocols and services associated to the aggressive communication market, creates strong dependencies from each PVN to the adopted implemented technology.

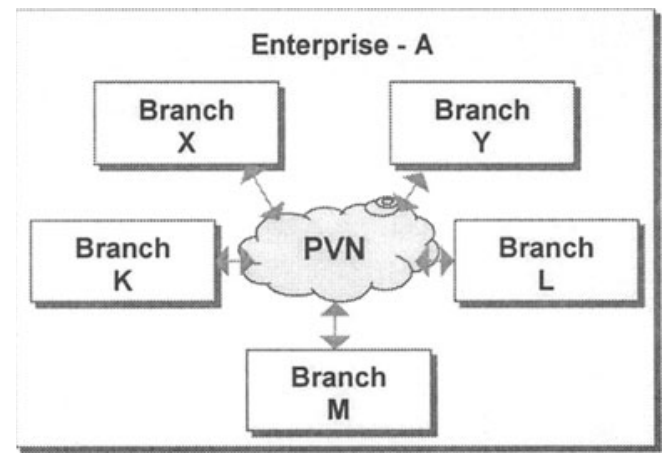

Figure 2 - Communication infrastructure linking different enterprise branches

When required to establish communication links between different companies the problem relies on the available technological solutions to support it. They may involve different communication service providers and incompatible communications technologies, creating a heterogeneous space guided by complementary policies. There are companies that establish their PVN over X25 synchronous communication lines, others have already adopted high-speed ISDN lines and, more recently with the emergence of the Internet, another infrastructure has been established to support private networks. To support a communication with all partners, an enterprise may have to adapt its communication infrastructure according to all the partners, what is not a realistic direction. Another alternative is to establish a communication agreement with Value Added Networks (VAN). The added value of the VANs is to create a uniform communication infrastructure for all the enterprises that need to exchange electronic information among them. The adaptation to the specific communication technology of each enterprise is a VAN entity responsibility. 


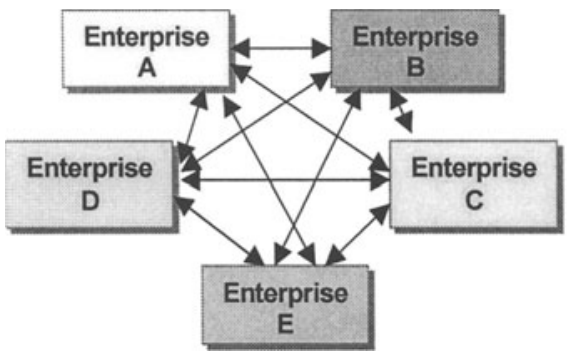

a)

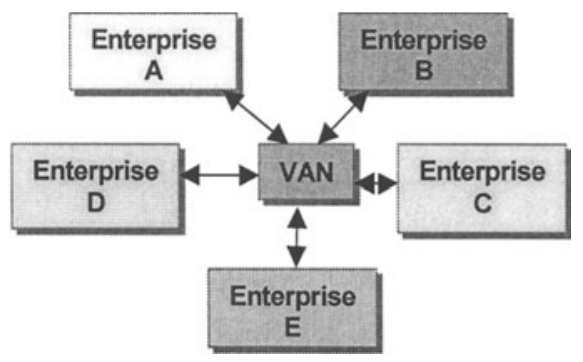

b)

Figure 3 - Communication among enterprises: a) direct links; b) through a VAN

In fact, enterprises always face complex problems when deciding to go toward an electronic information exchange. The part a) of Figure 3 shows a scenario where different enterprises establish their own links between enterprises. Considering the complex infrastructure each enterprise has to manage to support such connections, this scenario is not a good one. This complexity leads to the emergence of the VANs, part b) of Figure 3, where all the enterprises have only one interlocutor, the VAN communication service provider.

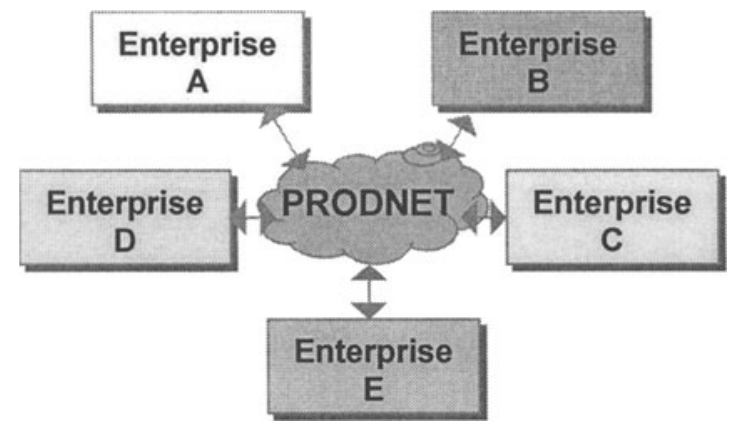

Figure 4 - Communication infrastructure linking different enterprises

However, the existence of a heavy infrastructure to plug such enterprise heterogeneity creates added costs, difficult to be accepted by SMEs. In this direction, the PRODNET infrastructure would play, to some extent, a normalization role offering different enterprises a uniform communication infrastructure. In addition, the proposed infrastructure might be opened and able to incorporate almost all the legated communication resources, if already available in the enterprise. This openness and flexibility aims to create a general adopted infrastructure, easy to maintain and, as it is able to operate over the Internet, the costs would be positioned according the target market - SME.

Therefore, the main characteristics required to be present in the PRODNET communication infrastructure are presented in the next point. 


\section{Legal implications of the electronic information exchange}

There is an intensive discussion going on about the laws that will govern all issues related with security in communication and information privacy.

Some governments are positioned in the most intransigent side, forbidding any form of encryption during information transportation. The main argument to behave like this is related with national security issues. National security agencies need to wiretap communications to survey its content for criminal investigations or to maintain preventive surveillance. As an example, the French government used to be a paradigmatic example of a country that has restrictive regulations on encryption, classifying ciphered communications and respective equipment in the category of weapons. Nevertheless, some governments are pointing to establish that specific utilization of encryption should be outside any special restriction framework; the Portuguese Government falls into this group.

There are several initiatives conducting the definition of a set of laws regulating the utilization of cryptographic techniques to guarantee confidentiality, integrity and authentication in opened spaces. Different governments are preparing new or refined legislation to deal with this new scenario, required by the global information infrastructure. There are four main concerns when dealing with encryption regulations/laws:

- governments want to maintain control over the information stored and exchanged, in order to protect citizens against terrorism and other form of attacks;

- persons want to guarantee privacy of their stored and exchanged information; even for national security utilization, as it is used in almost all countries, the access should be restricted in front of a court order;

- non-repudiation or electronic agreement enforcement needs to be guaranteed by mechanisms with legal support; all litigant parts should be able to prove their believes in the court;

- encryption used by specialized frameworks to guarantee privacy, integrity and authentication should be outside any legal restriction; it is not reasonable that private information exchanged or stored in any place, be subject to permanent observation by any other entity than the owner.

The PRODNET infrastructure might be able to comply with the legal framework each company is mandatory to follow. It is mandatory for the communication infrastructure to offer trust mechanisms creating in users the required confidence.

\section{Openness of the communication infrastructure}

An important characteristic for any communication infrastructure relies on its openness. The enterprises want to adopt technologies able to incorporate their assets and to offer technology provider independence.

The strategy behind the PRODNET communication infrastructure is to offer the ability to incorporate new communication resources in order to increase availability. Other important aspect relies on the adoption of standards to manage different 
aspects of the communication infrastructure. As an example of such approach, the X509 certificates were adopted, making possible the incorporation of certificates generated by other systems. Besides, a simple and clear application programming interface makes possible to enterprise applications to access the communication infrastructure. This facet involves the public interface of the PRODNET kernel described in detail in the next chapters.

\section{CONCLUSIONS}

The communication infrastructure has been one of the main obstacles to a faster growing of the electronic relations among enterprises to support business document flows.

The emergence of the Internet technology suit has created new expectations for a more effective and high quality communication infrastructure. However, the basic requirements for any communication infrastructure are not easy to provide, not from the technology itself, but because there are a large number of technological solutions competing to the same objectives. For SME companies it is difficult to make large investments creating an enterprise-networking infrastructure, able to cooperate with the technologies installed in all the partners. As an example, the adoption of X25 and X400 are confined to large enterprise or even governmental organizations. Its implantation involves costs not only at installation phase but also during the operation. On the other hand, an increasing utilization of the Internet protocol suit has opened new perspectives for SME, based on the fact that a general adoption of a technology decreases the prices and increases availability. The large number of companies with Internet access falls in this scenario.

Therefore, the adoption of the Internet as the basic communication infrastructure was established for PRODNET communication infrastructure. Nevertheless, considering the necessary interoperability with legated technologies, other communication resources may be integrated, increasing the availability and the reutilization of legated systems. The adoption of the Internet makes imperative the implementation of added security mechanisms and policies, in order to offer to each enterprise, connected to the PRODNET infrastructure, a trusted communication infrastructure. Even if some mechanisms related with security are under political decisions, like the establishment of a certification framework, the need for Trusted Third Parties playing the role of electronic notaries, from many others, is being discussed. The PRODNET communication infrastructure should be aware of those trends, in order to comply with the legal aspects of the electronic information exchanges.

\section{Acknowledgements}

This work was done in the context of the PRODNET II project, partially funded by the European Commission. The authors thank the valuable contributions from the consortium partners: UNL (P), CSIN (P), UvA (NL), UFSC (BR), UNINOVA (P), MIRALAGO (P), HERTEN (BR), LICHEN (F), ProSTEP(D) and ESTEC (P). 


\section{REFERENCES}

1. Camarinha-Matos, L. M., C.P. Lima and L. Osório. The PRODNET platform for Production Planning and Management in Virtual Enterprises, Proceedings of ICE'97- Int. Conference on Concurrent Enterprising, Nottingham, United Kingdom, 1997.

2. Cheswick W., Bellovin S. Firewalls and Internet Security, Addison-Wesley, 1996.

3. Coulouris G., Dollimore J., Kindberg T. Distributed Systems, Concepts and Design. AddisonWesley, 1994.

4. Dowd K. Getting Connected, O'Reilly \& Associates, 1996.

5. Jamsa K., Cope K. Internet Programming. Jamsa Press, 1995.

6. Macgregor R. S., Aresi A., Siegert A. WWW.Security, How to Build a Secure World Wide Web Connection, IBM, Prentice Hall PTR, 1996.

7. Osório, A. Luís; Gibon, Pierre; Barata M. Martins (1998) - Secure Electronic Commerce in Virtual Enterprises of SMEs, presented at the international conference BASYS'98, 27-28 August 1998 in Prague and published by Klower.

8. Schneier Bruce. Applied Cryptography. 2nd edition, John Wiley \& Sons, 1996.

9. Stross C. The Web Architect's Handbook. Addison-Wesley, 1997.

10. Tanenbaum Andrew S. Distributed Operating Systems, Prentice-Hall, 1994

11. Willmott R. Secure Inter-organisational Electronic Messaging, Draft Memorandum of Understanding, 1998.

12. Willmott R. Secure Inter-organisational Electronic Messaging, Framework, 1998. 